\title{
A Note on the Correspondence Pattern for Ordinary People
}

\author{
Feng Wang and Xianyuan $\mathrm{Wu}^{*}$ \\ School of Mathematical Sciences, Capital Normal University, Beijing, 100048, P. R. China \\ ${ }^{*}$ Corresponding author
}

\begin{abstract}
This paper focuses on the problem of modeling the correspondence pattern for ordinary people. Suppose that letters arrive at a rate $\lambda$ and answered at a rate $\mu$. Furthermore, we assume that, for a constant $T$, and the remains are answered in last in first out order. Let $W_{n}$ be the waiting time of the $n$-th answered letter. It is proved that $W_{n}$ converges weekly to $W_{T}$, a non-negative random variable which possesses a density with power-law tail when $\lambda=\mu$ and with exponential otherwise. Note that this may provide a reasonable explanation to the phenomenon reported by Oliveira and Barabási in [16].
\end{abstract}

Keywords-M/M/1 queue; last in first out; waiting time; tail probability; Laplace transforms

\section{INTRODUCTION}

In 2005, Oliveira and Barabási [16] reported their research results on the correspondence patterns of Darwin and Einstein: during their lifetimes, Darwin and Einstein answered a fraction of letters they received (the over-all response rate being 0.32 and 0.24 , respectively), and the distributions of response times to letters are both well approximated with a power-law tail that has an exponent $\alpha=3 / 2$. The classical $M / M / 1$ process [10, Section 2.2], which assumes that letters arrive at a rate $\lambda$ and are answered at a rate $\mu$, can be used to model their correspondence patterns. Under some service discipline, the waiting-time density of the $\mathrm{M} / \mathrm{M} / 1$ process may follow $\mathrm{f}(\mathrm{t})$ $t^{-\frac{3}{2}} \exp \left(-t / t_{0}\right)$ for $\lambda \leq \mu$ (see [1]), which predicts a power-law waiting time for the critical regime $\lambda=\mu$ when $t_{0}=\infty$. However, by the response rates 0.32 and 0.24 , we have $\lambda>$ $\mu$ and this places the model in the supercritical regime, where a finite fraction of letters is never answered. Oliveira and Barabási pointed out in [16] that numerical simulations indicate that in this supercritical regime the waiting-time distribution of the responded letters also follows a power law with exponent $\alpha=3 / 2$.

Clearly, [16] proposed such questions on modeling the correspondence patterns of human being: how do the ordinary people prioritize the correspondence in need of a response? And does a usual priority principle really lead to a power-law waiting time in the supercritical regime? To partially answer the above questions, or at least, to provide some useful evidence for understanding the above questions, in the present paper, we introduce a special queueing system with a service discipline, which corresponds an usual priority principle of ordinary people, and then study the waiting time for served customers, especially in the supercritical regime.
Now, let's consider the usual M/M/1 queue, the simplest queueing model used in practice. Suppose that the arrivals occur in a Poisson process with rate $\lambda$ and the service times of the unique server have an exponential distribution with parameter $\mu$. We put the service discipline as follows: for some fixed $\mathrm{T}>0$, a customer leaves the queue when his waiting time exceeds $\mathrm{T}$; the remains are served on the last in first out principle, namely, the principle serves customers one at a time, and the customer with the shortest waiting time will be served first. Note that our model is a queueing system with impatient customers, which was first proposed by Barrer [3, 4], then followed by $[8,12,13,14]$ etc.. For details on queues with impatience, one may refer to [18] and the references therein.

Assume that at time 0 , a customer (the 0 -th customer) arrives and the queueing system begins to work. Let $\left\{X_{n}: \mathrm{n} \geq\right.$ $1\}$ be an i.i.d sequence of exponential distribution random variables with parameter $\lambda$, independent of $\left\{X_{n}: n \geq 1\right\}$, $\left\{Y_{n}: \mathrm{n} \geq 1\right\}$ be another i.i.d sequence of exponential distribution random variables with parameter $\mu$. Let $S_{n}^{X}=$ $\sum_{i=1}^{n} X_{i}$ and $S_{n}^{Y}=\sum_{i=1}^{n} Y_{i}$. In our system, the n-th customer arrives at time $S_{n}^{X}$.

Now, fix some $\lambda, \mu>0$ and $0<\mathrm{T} \leq \infty$. Let $N_{t}$ be the queue length, namely, the total number of customers in the system at time t. It is clear that $\left\{N_{t}: \mathrm{t} \geq 0\right\}$ is no longer a Markov process for any $0<T<\infty$; and in the case of $\mathrm{T}=\infty,\left\{N_{t}: \mathrm{t} \geq\right.$ 0 \} degenerates to the classical birth-death process with birth rate $\lambda$, and death rate $\mu$. For any $\mathrm{n} \geq 0$, Denote by $D_{n}$ the waiting time of the n-th customer, namely, $D_{n}$ is the usual waiting time when he is finally served before his waiting time exceeds T, otherwise, $D_{n}=\infty$. By the setting given in the last paragraph, one has $N_{0}=1$ and $D_{0}=0$. Let

$$
\tau=\tau(\lambda, \mu, T)=\inf \left\{n \geq 1: D_{n}=0\right\} .
$$

Denote $q_{k}=P(\tau=k), k \geq 1$, and let $\mathrm{M}=\mathrm{M}(\lambda, \mu, \mathrm{T})=$ $\mathrm{E}(\tau)$ be the expectation of $\tau$.

First of all, we have the following proposition on $\mathrm{E}(\tau)$.

Proposition 1.1 i) for any $\lambda, \mu>0$ and $0<T \leq \infty$, we have

$$
M=M(\lambda, \mu, T)=E(\tau)<\infty
$$

ii) for any given $\mu>0, M=M(\lambda, \mu, T)$ increases in $\lambda$ and T. Furthermore, for any given $\lambda$ and $\mu$, 


$$
\lim _{T \rightarrow \infty} M(\lambda, \mu, T)=M(\lambda, \mu) \begin{cases}<\infty, & \text { if } \lambda<\mu, \\ =\infty, & \text { if } \lambda \geq \mu .\end{cases}
$$

Let $\bar{D}_{n}=0$ be the number of customers who have already been in the queue when the n-th customer arrives. Then, almost surely, $D_{n}=0$ if and only if $\bar{D}_{n}=0$, and $\tau$ is the first return time of state 0 for process $\left\{\bar{D}_{n}: n \geq 0\right\}$.

By i) of Proposition 1.1 and a renewal limit theorem for general stochastic processes (see [5] or [9, Section 8, Chapter 11]), the limit

$$
\lim _{n \rightarrow \infty} P\left(D_{n}=0\right)=\lim _{n \rightarrow \infty} P\left(\bar{D}_{n}=0\right) \text { exists }
$$

and will be proved to be 1/M (see Lemma 2.1 in Section 2).

We point out that, for any $0<\mathrm{T} \leq \infty$, by i) of Proposition 1.1 and the renewal limit theorem in [5], our system tends to statistical equilibrium, namely the queue length $N_{t}$ converges in distribution to a $Z_{+}$-valued random variable $\mathrm{N}$ as $\mathrm{t} \rightarrow \infty$.

Let $I_{1}$ be the modified Bessel function of first kind for real and positive t, see [17], given by

$$
I_{1}(t)=\sum_{m=0}^{\infty} \frac{1}{m !(m+1) !}\left(\frac{t}{2}\right)^{2 m+1} \sim \frac{e^{t}}{\sqrt{2 \pi t}}
$$

as $t \rightarrow \infty$. Note that for two functions $a(t)$ and $b(t), a(t) \sim b(t)$, $\mathrm{t} \rightarrow \infty$ means that $\lim _{t \rightarrow \infty} \frac{a(t)}{b(t)}=1$.

Now, we can state our main theorem as follows.

Theorem 1.2 For any $\lambda, \mu>0$ and $0<T \leq \infty, W_{n}$, the waiting time of the $n$-th served customer, converges in distribution to a non-negative random variable $W_{T}$. Furthermore, the distribution function $F_{T}$ of $W_{T}$ satisfies $F_{T}(x)=0$, for $x<0, F_{T}(x)=1$, for $x>T$, and

$$
F_{T}(x)=\frac{1}{C(T)}\left[\frac{1}{M}+\frac{1}{\rho \vee 1}\left(1-\frac{1}{M}\right) \int_{0}^{x} f_{\rho}(t) d t\right]
$$

for $0 \leq x \leq T$, where $C(T)$ is the normalization constant, $\rho=$ $\frac{\lambda}{\mu}$ and

$$
f_{\rho}(t)=\sqrt{\rho \vee \rho^{-1}} \frac{1}{t} e^{-(\lambda+\mu) t} I_{1}(2 t \sqrt{\lambda \mu}), t>0 .
$$

Remark 1.1 The function $f_{\rho}$ given in (6) is the probability density of $D$, the length of the busy period in the classical $M / M / 1$ system when $\rho \leq 1$, and is the conditional probability density of $D$ conditioned on $D<\infty$ when $\rho>1$. Note that in case of $\rho>1, P(D<\infty)=\rho^{-1}$.

Remark 1.2 For any large $0<T \leq \infty, f_{T}(t)$, the density of $W_{T}$ has a power-law tail with exponent $\alpha=3 / 2$ in the critical regime $\lambda=\mu$ as $t \lambda T$. In both subcritical and supercritical cases, $f_{T}(t)$ decays exponentially fast.

But, what are the correspondence patterns of Darwin and Einstein? Numerical simulations indicate that they always keep themselves in the critical regime. A reasonable explanation may be the following: letters arrive according to a Poisson process with rate $\lambda=\lambda_{1}+\lambda_{2}>\mu$, where $\lambda_{1}<\mu$ is the rate of letters heard from friends and family members, $\lambda_{2}$ is the rate of letters heard from the strangers. As the most distinguished scientists in their research fields, Darwin and Einstein received too many letters from the strangers, so they had to ignore such a received letter with probability $1-(\mu-$ $\left.\lambda_{1}\right) / \lambda_{2}$, such that, in their eyes, letters arrived according to a Poisson process with rate $\lambda_{1}+\lambda_{2} \times \frac{\mu-\lambda_{1}}{\lambda_{2}}=\mu$.

\section{PROOFS}

In this section we will prove Proposition 1.1 and Theorem 1.2. Before we give a proof to Proposition 1.1, we introduce an algorithm to obtain $\tau$.

Given $\lambda, \mu$ and T, let $S_{n}^{X}$ and $S_{n}^{Y}$ be defined as in Section 1 and let $\bar{S}_{n}^{X}=S_{n}^{X}+\mathrm{T}$. For any integer $\mathrm{k} \geq 1$, define the random index set $N_{k}$ as

$$
N_{k}:=\left\{n: S_{k-1}^{Y} \leq S_{n}^{X}<S_{k}^{Y}\right\}, \text { where } S_{0}^{Y}=0 .
$$

Let $\bar{N}_{k}=\bigcup_{i=1}^{k} N_{i}$ and write $n_{k}$ as the largest element in $\bar{N}_{k}$ when $\bar{N}_{k} \neq \phi$.

Now we will start a procedure to define a sequence of index sets $\left\{J_{k}: \mathrm{k} \geq 1\right\}$, we write $j_{k}$ as the largest element of $J_{k}$ when $J_{k} \neq \phi$. Let $J_{1}=\left\{n \in N_{1}: \bar{S}_{n}^{X}>S_{1}^{Y}\right\}$. If $J_{1} \neq \phi$, then let $J_{1}^{\prime}=J_{1} \backslash\left\{j_{1}\right\}$ and define $J_{2}=\left\{n \in J_{1}^{\prime} \cup N_{2}: \bar{S}_{n}^{X}>S_{2}^{Y}\right\}$; otherwise, the procedure is stopped. By induction, for any $\mathrm{k} \geq$ 2 , if $J_{k}$ has already been defined and $J_{k} \neq \phi$, then let $J_{k}^{\prime}=$ $J_{k} \backslash\left\{j_{k}\right\}$ and define $J_{k+1}=\left\{n \in J_{k}^{\prime} \cup N_{k+1}: \bar{S}_{n}^{X}>S_{k+1}^{Y}\right\}$; otherwise, the procedure is stopped. Let

$$
\tau_{1}=\inf \left\{k \geq 1: J_{k}=\phi\right\},(\text { set inf } \phi=\infty) .
$$

Then

$$
\tau= \begin{cases}n_{\tau_{1}}+1, & \text { if } \tau_{1}<\infty \\ \infty, & \text { otherwise }\end{cases}
$$

Proof of i) of Proposition 1.1. Suppose that $0<\mathrm{T}<\infty$. For any $\mathrm{k} \geq 1$, let $A_{k}$ be the event that $Y_{k} \geq T$ and $N_{k}=\phi$. Recalling that the random point set $\left\{S_{n}^{X}: \mathrm{n} \geq 1\right\}$ forms a one dimensional Poisson point process in $R_{+}$, we know that $\left\{A_{k}\right\}$ are mutually independent and, for any $\mathrm{k} \geq 1$,

$$
\begin{aligned}
p_{0}:= & P\left(A_{k}\right)=\int_{T}^{\infty} P\left(N_{k}=\phi \mid Y_{k}=t\right) \mu e^{-\mu t} d t \\
& =\int_{T}^{\infty} e^{-\lambda t} \mu e^{-\mu t} d t=\frac{\mu}{\lambda+\mu} e^{-(\lambda+\mu) T}
\end{aligned}
$$

Let $\tau_{2}=\inf \left\{k \geq 1: A_{k}\right.$ occurs $\}$. First, we point out that $\tau_{2}$ is a geometry distribution random variable with parameter $p_{0}$, hence, we have

$$
\mathrm{E}\left(\tau_{2}\right)=\frac{1}{p_{0}}=\frac{\lambda+\mu}{\mu} e^{(\lambda+\mu) T} .
$$


Second, by (7), we have $\tau_{1} \leq \tau_{2}$ and $n_{\tau_{1}} \leq n_{\tau_{2}}$. Denote by $h_{k}$ the cardinality of $N_{k}$, then

$$
n_{\tau_{2}}=\sum_{k=1}^{\tau_{2}} h_{k}
$$

and

$$
\begin{gathered}
\mathrm{E}\left(n_{\tau_{2}}\right)=\sum_{n=1}^{\infty} E\left(n_{\tau_{2}} \mid \tau_{2}=n\right) P\left(\tau_{2}=n\right) \\
\quad=\sum_{n=1}^{\infty} \sum_{k=1}^{n} E\left(h_{k} \mid \tau_{2}=n\right) P\left(\tau_{2}=n\right)
\end{gathered}
$$

Note that $\left\{\tau_{2}=\mathrm{n}\right\}=\bigcap_{k=1}^{n-1} A_{k}^{C} \cap A_{n}$, then $\mathrm{E}\left(h_{n} \mid \tau_{2}=\mathrm{n}\right)=0$ and, for any $1 \leq \mathrm{k} \leq \mathrm{n}-1$,

$$
\begin{aligned}
\mathrm{E}\left(h_{k} \mid \tau_{2}=\mathrm{n}\right) & =\mathrm{E}\left(h_{k} \mid A_{k}^{C}\right)=\mathrm{E}\left(h_{k} \mid h_{k}>0\right) P\left(h_{k}>0 \mid A_{k}^{C}\right) \\
& \leq \mathrm{E}\left(h_{k} \mid h_{k}>0\right)=\frac{E\left(h_{k}\right)}{P\left(h_{k}>0\right)}
\end{aligned}
$$

where the first equality comes from the fact that $h_{k}$ is independent of $A_{k}$ for any $k^{\prime} \neq k$. Clearly,

$$
\mathrm{E}\left(h_{k}\right)=\int_{0}^{\infty} E\left(h_{k} \mid Y_{k}=t\right) \mu e^{-\mu t} d t=\int_{0}^{\infty} \lambda t \mu e^{-\mu t} d t=\frac{\lambda}{\mu}
$$

and

$$
\begin{aligned}
\mathrm{P}\left(h_{k}>0\right) & =\int_{0}^{\infty} P\left(h_{k}>0 \mid Y_{k}=t\right) \mu e^{-\mu t} d t \\
& =\int_{0}^{\infty}\left(1-e^{-\lambda t}\right) \mu e^{-\mu t} d t=\frac{\lambda}{\lambda+\mu}
\end{aligned}
$$

Thus, by (9), (10) and then by (8),

$$
\begin{aligned}
\mathrm{E}(\tau) & =\mathrm{E}\left(n_{\tau_{1}}+1\right) \leq \mathrm{E}\left(n_{\tau_{2}}+1\right) \\
& \leq \frac{\lambda+\mu}{\mu} \sum_{n=1}^{\infty}(n-1) P\left(\tau_{2}=n\right)+1 \\
& \leq \frac{\lambda+\mu}{\mu} E\left(\tau_{2}\right)<\infty .
\end{aligned}
$$

Proof of ii) of Proposition 1.1. Given $\mu$ and T. To see $M(\lambda, \mu, T)$ increases in $\lambda$, one only need to notice the fact that two Poisson processes with parameters $\lambda_{1}$ and $\lambda_{2}, \lambda_{1}<\lambda_{2}$, respectively can be coupled together in such a way that all arrivals of the former forms a part arrivals of the later. Note that this follows from the infinitely divisible property of Poisson distribution [11]. For details on coupling technique in probability theory, please refer to [15]. Then, the corresponding monotonicity follows from (7), the definition of $\tau_{1}$.

For given $\lambda$ and $\mu$, the fact that $M(\lambda, \mu, T)$ increases in $\mathrm{T}$ follows directly from the definition of $\tau_{1}$.
Now we are in the way to prove (3), note that the $\lambda \geq \mu$ part of (3) only need to be proved for $\lambda=\mu$, the case of $\lambda>$ $\mu$ follows from the monotonicity in $\lambda$ proved above.

We first declare that $M(\lambda, \mu, \infty)<\infty$ for $\lambda<\mu$ and $=\infty$ for $\lambda=\mu$. In fact, in case of $T=\infty$, our system degenerates to the classical M/M/1 queue, the standard argument of the birthdeath process (see $[2,6]$ ) tells us that

$$
P(\tau(\lambda, \mu, \infty)<\infty)=1 \text { for } \lambda \leq \mu,
$$

and

$$
\mathrm{M}(\lambda, \mu, \infty)=\mathrm{E}(\tau(\lambda, \mu, \infty))\left\{\begin{array}{l}
<\infty, \text { for } \lambda<\mu \\
=\infty, \text { for } \lambda=\mu
\end{array}\right.
$$

Second, by (11) and the algorithm we have used to obtain $\tau$, we have $\tau(\lambda, \mu, T)$ increases in $T$, and

$$
\lim _{T \rightarrow \infty} \tau(\lambda, \mu, T)=\tau(\lambda, \mu, \infty), \text { a.s. }
$$

Thus, (3) follows from the monotone convergence theorem.

Before we give a proof to Theorem 1.2, we have to prove the following lemma. Note that this lemma plays the key role in the proof of Theorem 1.2.

Lemma 2.1 Suppose that $\lambda, \mu>0$, and $0<T \leq \infty$, then

$$
\lim _{n \rightarrow \infty} P\left(D_{n}=0\right)=\frac{1}{M(\lambda, \mu, T)}
$$

Proof. In case of $\mathrm{T}=\infty$, we are dealing with a classical $\mathrm{M} / \mathrm{M} / 1$ system, (12) follows from a standard argument for birth-death process $[2,6]$. Now, we suppose $0<T<\infty$.

Let $P_{00}^{(n)}=P\left(D_{n}=0\right), n \geq 0$. Then $P_{00}^{(0)}=1$, and for any $\mathrm{n} \geq 1$,

$$
P_{00}^{(n)}=\sum_{k=1}^{n} q_{k} P_{00}^{(n-k)},
$$

where $q_{k}=P(\tau=k)$. (13) indicates that the sequence $\left\{P_{00}^{(n)}: \mathrm{n} \geq 0\right\}$ is iteratively determined by $\left\{q_{k}: k \geq 1\right\}$ and its initial value $P_{00}^{(0)}=1$.

Provided (4) and (13), the lemma may follow from a standard argument on generation function and the Abel's Theorem (see [7, page 12]). Here, we prefer to give it a probabilistic proof, one will see that our proof mainly depends on (13), and (4) is only a consequence of it.

By the basic theory on discrete-time Markov chains [7, Section 1.2], to prove (12), it suffices to construct a $Z_{+}$-valued discrete-time Markov chain $\left\{\xi_{n}: \mathrm{n} \geq 0\right\}$ such that $\left\{\xi_{n}\right\}$ is ergodic and

$$
f_{00}^{(k)}:=P\left(\tau_{0}^{+}=k \mid \xi_{0}=0\right)=q_{k}, k \geq 1,
$$


where $\tau_{0}^{+}=\inf \left\{n \geq 1: \xi_{0}=0\right\}$ is the first return time of state 0 . Note that ergodic means irreducible, aperiodic and positive recurrent as usual.

To this end, we give the following transition matrix $\mathrm{P}=$ $\left(P_{i j}\right)$ to $\left\{\xi_{n}\right\}$ :

$$
P_{i j}= \begin{cases}\frac{q_{i+1}}{1-\sum_{k=1}^{i} q_{k}}, & j=0 ; \\ 1-P_{i 0}, & j=i+1 ; \\ 0, & \text { else. }\end{cases}
$$

It is straightforward to check that $\left\{\xi_{n}\right\}$ with the above transition matrix $\mathrm{P}$ is ergodic and satisfies (14). Thus we finish the proof of the lemma.

Proof of Theorem 1.2. For any $0 \leq \mathrm{x} \leq \mathrm{T}$, one has

$$
\begin{aligned}
& \mathrm{P}\left(D_{n} \leq \mathrm{x}\right) \\
= & \mathrm{P}\left(D_{n}=0\right)+\mathrm{P}\left(D_{n} \leq \mathrm{x} \mid D_{n}>0\right) \mathrm{P}\left(D_{n}>0\right) .
\end{aligned}
$$

First of all, $D_{n}>0$ if and only if, at $S_{n}^{X}$, the arrival time of the n-th customer, the unique server is occupied, namely, $\bar{D}_{n}>$ 0 . It is clear that, on the last in first out principle, if $\bar{D}_{n}>0$, then $D_{n}$ does not depend on the exact value of $\bar{D}_{n}$. Second, for any $0<x \leq T, D_{n} \leq x$ means that all customers arrived after, but were served before the $n$-th one are finally served in time $\mathrm{x}(\leq \mathrm{T})$. In other word, the $\mathrm{n}$-th customer finally gets into the server after a whole busy period finishes in time $\mathrm{x}$. Hence, by the memoryless property of the exponential distribution, for any $0<x \leq T$ and for any $\mathrm{n} \geq 1$,

$$
P\left(D_{n} \leq x \mid D_{n}>0\right)=P(D \leq x)=: F_{D}(x),
$$

where $D$ is the length of the busy period of the classical M/M/1 system. Note that, in our setting, $\mathrm{D}=S_{\tau_{3}}^{Y}$ with

$$
\tau_{3}=\inf \left\{n: S_{n}^{Y}<S_{n}^{X}\right\}
$$

As calculated in [19],

$$
\begin{gathered}
F_{D}(x)=\sum_{m=1}^{\infty} \int_{0}^{x} e^{-\lambda t} \frac{(\lambda t)^{m-1}}{m !} d F_{S_{m}^{Y}}(t) \\
=\sum_{m=0}^{\infty} \int_{0}^{x} \frac{\mu(t \sqrt{\lambda \mu})^{2 m}}{m !(m+1) !} e^{-(\lambda+\mu) t} d t
\end{gathered}
$$

where $F_{S_{m}^{Y}}$ is the distribution function of $S_{m}^{Y}$.

In case of $\lambda \leq \mu$, by (16), we have $\mathrm{P}(\mathrm{D}<\infty)=\mathrm{P}\left(\tau_{3}=\right.$ $\infty)=1$, so $F_{D}$ is a probability distribution function and

$$
f_{D}(t):=F_{D}^{\prime}(t)=\sqrt{\frac{\mu}{\lambda}} \frac{1}{t} e^{-(\lambda+\mu) t} I_{1}(2 t \sqrt{\lambda \mu})
$$

is a probability density.
To finish the proof of the theorem, we will introduce $\Gamma$, the Laplace transform of $F_{D}$ defined by the following LebesgueStieltjes integration

$$
\Gamma(\mathrm{s})=\int_{0}^{\infty} e^{-s t} d F_{D}(t), \quad s>0 .
$$

It is calculated directly that (see [19, Eq. (51)]),

$$
\Gamma(s)=\frac{1}{2 \lambda}\left[\lambda+\mu+s-\sqrt{(\lambda+\mu+s)^{2}-4 \lambda \mu}\right] .
$$

Clearly, $\Gamma$ can be uniquely inverted to give the probability distribution function $F_{D}$ in case of $\lambda \leq \mu$.

In case of $\lambda>\mu$, by symmetry and the linearity of the (inverse) Laplace Transform, the following

$$
\tilde{\Gamma}(\mathrm{s})=\rho \Gamma(\mathrm{s})=\frac{1}{2 \mu}\left[\lambda+\mu+s-\sqrt{(\lambda+\mu+s)^{2}-4 \lambda \mu}\right]
$$

can be inverted to give the following probability distribution function

$$
\tilde{F}_{D}(x):=\rho F_{D}(x)=\frac{P(D \leq x)}{P(D<\infty)}=P(D \leq x \mid D<\infty),
$$

and $\tilde{f}_{D}=\rho f_{D}$ is the probability density corresponding to $\tilde{F}_{D}(x)$.

By (20), one has $\mathrm{P}(\mathrm{D}<\infty)=\rho^{-1}$ and $\widetilde{F}_{D}(x)$ is the conditional distribution function of $\mathrm{D}$ conditioned on $\mathrm{D}<\infty$.

Write $f_{D}$ and $\tilde{f}_{D}$ in the unified form $f_{\rho}$, which is given in (6). Then, by (15), the conditional distribution of $D_{n}$ conditioned on $D_{n} \leq T$ is

$$
\begin{aligned}
F_{n}(x) & :=P\left(D_{n} \leq x \mid D_{n} \leq T\right) \\
& =\frac{1}{C_{n}(T)}\left[\mathrm{P}\left(D_{n}=0\right)+\mathrm{P}\left(D_{n}>0\right) \frac{1}{\rho \vee 1} \int_{0}^{x} f_{\rho}(t) d t\right]
\end{aligned}
$$

for $0 \leq \mathrm{x} \leq \mathrm{T}$, where $C_{n}(T)$ is the normalization constant. The theorem follows immediately form (21) and Lemma 2.1.

\section{ACKNOWLEDGMENT}

Research supported in part by the Natural Science Foundation of China (under grants 11271356, 11471222, 11671275) and the Foundation of Beijing Education Bureau (under grant KM201510028002).

\section{REFERENCES}

[1] J. Abate and W. Whitt “ Assymptotics for M/G/1 low-priority waiting time tail probabilities”, Queueing Systems 25, 1997, pp 173-233

[2] W. J. Anderson “Continous-Time Markov Times”, Springer-Verlag, New York, 1991

[3] D. Y. Barrer “Queuing with impatient customers and indifferent clerks”, Oper. Res. 5, 1957, pp 644-649 
[4] D. Y. Barrer "Queuing with impatient customers and ordered services", Oper. Res. 5, 1957, pp 650-656

[5] V. E. Beneš (1962) "A 'renewal' limit theorem for general stochastic processes”, Ann. Math. Statist. 33, pp 98-113

[6] M.-F. Chen "From Markov Chains to Non-equilibrium Particle Systems", 2nd ed. World Scientific Publishing Company, Singapore, 2004

[7] M.-F. Chen and Y.-H. Mao "An Introduction to Random Processes”, (in chinese), Higher education press, 2007, ISBN 978-7-04-021778-0

[8] P. D. Finch "Deterministic customer impatience in the queueing system GI/M/1”, Biometrika 47(1,2) , 1960, pp 45-52

[9] W. Feller "An introduction to prabability theory and its applications", Vol. II, John Wiley \& Sons, Inc. 1971

[10] D. Gross, J. F. Shortle, J. M. Thompson and C. M. Harris "Fundamentals of Queueing Theory”, 4th ed., John Wiley \& Sons, Inc. Hoboken, New Jersey, 2008

[11] N. L. Johnson, S. Kotz and A. W. Kemp (1993) Univariate Discrete distributions 2nd ed., John Wiley \& Sons, Inc., ISBN 0-471-54897-9, p. 159

[12] O. M. Jurkevic "On the investigation of many-sever queueing system with bounded waiting time”, (in Russian) Izv. Akad. Nauk SSSR Techniceskaja Kibernetika 5, 1970, pp 50-58

[13] O. M. Jurkevic "On many-server systems with stochastic bounds for the waiting time", (in Russian) Izv. Akad. Nauk SSSR Techniceskaja Kibernetika 4, 1971, pp 39-46

[14] A. G. de Kok and H. C. Tijms "A queueing system with impatient customers”, J. Appl. Probab. 22, 1985, pp 688-696

[15] T. Lindvall "Lectures on the coupling method", John Wiley \& Sons, Inc., New York, 1992

[16] J. G. Oliveira and A.-L. Barabási "Darwin and Einstein correspondence patterns”, Nature 437, 2005, p. 1251

[17] F. W. J. Olver "Bessel Functions of Interger Order, in Handbook of Mathematical Functions with Formulas, Graphs, and Mathematical Tables”, edited by M. Abramowitz and I. A. Stegun, 10th printing. New York: Dover, 1972, pp 355-434

[18] K.-Zh. Wang, N. Li and Zh.-B. Jiang "Queueing system with impatient customers: A review”, 2010, DOI: 10.1109/SOLI.2010.5551611

[19] G.-H. Xu "Random Service Systems”, (in chinese), Science Press, 1980 